Supporting Information

\title{
Cyprosulfamide: Analysis of the herbicide safener and two of its degradates in surface water and groundwater from the Midwestern United States
}

\author{
Monica E. McFadden ${ }^{\dagger}, \dot{\dagger}$, Michelle L. Hladik ${ }^{\S, *}$ \\ $\dagger^{\dagger}$ Department of Civil and Environmental Engineering, University of Iowa, 4105 Seamans Center \\ for the Engineering Arts and Sciences, Iowa City, Iowa 52242, United States; $₫$ IIHR- \\ Hydroscience and Engineering, University of Iowa, 100 C. Maxwell Stanley Hydraulics \\ Laboratory, Iowa City, Iowa, 52242, United States; § U.S. Geological Survey, 6000 J Street, \\ Placer Hall, Sacramento, California, 95819, United States
}

*Corresponding Author e-mail address: mhladik@usgs.gov; phone number: 916-278-3183

Supporting information includes: Sampling site information, cartridge conditioning protocol, analytical methods, LOD calculation, results 


\section{METHOD DETAILS}

Sampling Sites: The American River is a clean, snowmelt-fed water source; available water quality data indicate high dissolved oxygen concentrations $(9.7 \mathrm{mg} / \mathrm{L})$, circumneutral $\mathrm{pH}(7.3)$, and low concentrations of nitrate $(<0.06 \mathrm{mg} / \mathrm{L})$, phosphorus $(0.005 \mathrm{mg} / \mathrm{L}$ as $\mathrm{P})$, and organic carbon $(1.5 \mathrm{mg} / \mathrm{L}) .{ }^{1}$ Both the Ulatis Creek and Iowa River watersheds are primarily agricultural and have higher DOC content than the American River. ${ }^{2-4}$

Cartridge Conditioning: SPE method optimization involved pre-conditioning solid-phase extraction cartridges with $10 \mathrm{~mL}$ of each solvent that was later used during elution. Table S1 shows each solvent used for cartridge washing and cartridge elution during SPE method optimization. Where more than one elution was performed, solvent fractions were collected separately.

Table S1: Wash and elution solvents used during SPE method optimization.

\begin{tabular}{|c|c|c|c|c|c|}
\hline & MAX Cartridge & MAX Cartridge & WAX Cartridge & HLB Cartridge & HLB Cartridge \\
\hline Wash 1 $(10 \mathrm{~mL})$ & Ethyl Acetate & Acetone & Acetone & Methanol & Acetone \\
\hline Wash 2 $(10 \mathrm{~mL})$ & Methanol & Dichloromethane & Dichloromethane & Water & Dichloromethane \\
\hline Wash $3(10 \mathrm{~mL})$ & Water & Water & Water & & Water \\
\hline Elution $1(10 \mathrm{~mL})$ & Ethyl Acetate & $1: 1$ Acetone/DCM & $1: 1$ Acetone/DCM & Methanol & $1: 1$ Acetone/DCM \\
\hline Elution 2 $(10 \mathrm{~mL})$ & Methanol & & & & \\
\hline
\end{tabular}

\section{Analytical methods}

For compound analysis with mass spectrometer, samples were analyzed on electrospray ionization positive mode, gas temperature $350{ }^{\circ} \mathrm{C}$, gas flow $10 \mathrm{~L} / \mathrm{min}$, capillary voltage $4,000 \mathrm{~V}$, nebulizer 40 psi. Data were collected in multiple-reaction-monitoring (MRM) mode using two transition ions (quantitation and verification), with the ratio of the quantitation transition ion to the verification transition ion not exceeding $+/-25 \%$.

Table S2: LC-MS/MS Mobile phase gradient

\begin{tabular}{|c|c|c|}
\hline Time $(\min )$ & $\%$ Acetonitrile & $\% 5 \mathrm{mM}$ Formic Acid in organic-free water \\
\hline 0 & 2 & 98 \\
\hline 2 & 2 & 98 \\
\hline 4 & 50 & 50 \\
\hline 7 & 50 & 50 \\
\hline 7.5 & 2 & 98 \\
\hline 12 & 2 & 98 \\
\hline
\end{tabular}


Table S3: Multiple Reaction Monitoring (MRM) Parameters

\begin{tabular}{|c|c|c|c|c|c|c|c|}
\hline Compound & $\begin{array}{c}\text { Precursor } \\
\text { lon }\end{array}$ & $\begin{array}{c}\text { Quantitation } \\
\text { lon }\end{array}$ & $\begin{array}{c}\text { Qualitative } \\
\text { lon }\end{array}$ & $\begin{array}{c}\text { Fragmentor } \\
\text { Voltage }\end{array}$ & $\begin{array}{c}\text { Quantitation } \\
\text { ion collision } \\
\text { energy (V) }\end{array}$ & $\begin{array}{c}\text { Qualitative } \\
\text { ion collision } \\
\text { energy (V) }\end{array}$ & $\begin{array}{c}\text { Retention } \\
\text { time (min) }\end{array}$ \\
\hline Cyprosulfamide & 375.1 & 135 & 121 & 122 & 20 & 20 & 6.4 \\
\hline $\begin{array}{c}\text { Cyprosulfamide } \\
\text { desmethyl }\end{array}$ & 361.09 & 121 & 93 & 93 & 24 & 50 & 6.0 \\
\hline $\begin{array}{c}\text { N-cyclopropyl-4- } \\
\text { sulfamoylbenzamide }\end{array}$ & 241.07 & 184 & 75 & 64 & 4 & 50 & 5.3 \\
\hline
\end{tabular}

\section{Lower Limit of Detection Calculation:}

Table S4: Instrument limit of detection (iLOD) and Method limit of detection (mLOD) for cyprosulfamide and its degradates

\begin{tabular}{|l|c|c|}
\hline & iLOD $\left(\mathrm{ng} \mathrm{mL}^{-1}\right)$ & $\mathrm{mLOD}\left(\mathrm{ng} \mathrm{L}^{-1}\right)$ \\
\hline Cyprosulfamide & 0.00108 & 0.216 \\
\hline Cyprosulfamide desmethyl & 0.00034 & 0.068 \\
\hline N-cyclopropyl-4-sulfamoylbenzamide & 0.00046 & 0.091 \\
\hline
\end{tabular}

\section{RESULTS}

Table S5: Cyprosulfamide and degradate recoveries on various solid-phase extraction cartridges

\begin{tabular}{|c|c|c|c|c|}
\hline Cartridge Type & Solvent & Cyprosulfamide & Cyprosulfamide Desmethyl & $\begin{array}{c}\text { N-Cyclopropyl-4- } \\
\text { sulfamoylbenzamide }\end{array}$ \\
\hline & & \% Recovery & \% Recovery & Recovery \\
\hline MAX & Ethyl Acetate + Methanol & 39 & 0 & 36 \\
\hline MAX & $50 / 50$ Ace/DCM & 77 & 0 & 13 \\
\hline WAX & $50 / 50$ Ace/DCM & 0 & 0 & 84 \\
\hline HLB & Methanol & 99 & 91 & 93 \\
\hline HLB & $50 / 50$ Ace/DCM & 88 & 0 & 86 \\
\hline
\end{tabular}

Table S6: Analyte endogenous concentrations in surface waters of varying DOC content, as a percentage of spiked concentration

\begin{tabular}{|l|l|l|l|}
\hline Source Water & $\begin{array}{l}\text { Cyprosulfamide (ng/L) } \\
\text { (\% of spiked } \\
\text { concentration) }\end{array}$ & $\begin{array}{l}\text { Cyprosulfamide } \\
\text { Desmethyl (ng/L) } \\
\text { (\% of spiked } \\
\text { concentration) }\end{array}$ & $\begin{array}{l}\text { N-Cyclopropyl-4- } \\
\text { sulfamoylbenzamide (ng/L) } \\
\text { (\% of spiked concentration) }\end{array}$ \\
\hline lowa River & $2.1(2.2 \%)$ & ND & $3.2(3.5 \%)$ \\
\hline Ulatis Creek & ND & ND & ND \\
\hline American River & ND & ND & ND \\
\hline
\end{tabular}


Table S7: Cyprosulfamide and degradate recoveries in American River and DI water.

\begin{tabular}{|c|c|c|c|c|c|}
\hline Sample & Solvent & Cyprosulfamide & $\begin{array}{l}\text { Cyprosulfamide } \\
\text { Desmethyl }\end{array}$ & $\begin{array}{c}\text { N-Cyclopropyl-4- } \\
\text { sulfamoylbenzamide }\end{array}$ & $\begin{array}{l}\mathrm{d}_{4} \text {-Imidacloprid } \\
\text { (Surrogate) }\end{array}$ \\
\hline & & \% Recovery & \% Recovery & $\%$ Recovery & \% Recovery \\
\hline $\begin{array}{l}\text { American } \\
\text { R. (\#1) }\end{array}$ & Methanol & 75 & 38 & 82 & 62 \\
\hline $\begin{array}{l}\text { American } \\
\text { R. (\#2) }\end{array}$ & Methanol & 62 & 26 & 71 & 55 \\
\hline $\begin{array}{l}\text { American } \\
\text { R. (\#3) }\end{array}$ & Methanol & 34 & 37 & 39 & 43 \\
\hline $\begin{array}{l}\text { American } \\
\text { R. }(\# 4)\end{array}$ & Acetone/DCM & 15 & 0 & 66 & 82 \\
\hline $\begin{array}{l}\text { American } \\
\text { R. (\#5) }\end{array}$ & Acetone/DCM & 18 & 2 & 84 & 90 \\
\hline $\begin{array}{l}\text { American } \\
\text { R. (\#6) }\end{array}$ & Acetone/DCM & 30 & 1 & 104 & 100 \\
\hline $\begin{array}{l}\text { American } \\
\text { R. }(\# 4)\end{array}$ & Methanol & 42 & 37 & 17 & 6 \\
\hline $\begin{array}{l}\text { American } \\
\text { R. (\#5) }\end{array}$ & Methanol & 58 & 47 & 16 & 5 \\
\hline $\begin{array}{l}\text { American } \\
\text { R. (\#6) }\end{array}$ & Methanol & 56 & 49 & 4 & 1 \\
\hline DI (\#1) & Acetone/DCM & 95 & 1 & 83 & 89 \\
\hline DI (\#2) & Acetone/DCM & 96 & 1 & 82 & 95 \\
\hline DI (\#3) & Acetone/DCM & 99 & 0 & 93 & 94 \\
\hline DI (\#1) & Methanol & 6 & 71 & 9 & 3 \\
\hline DI (\#2) & Methanol & 10 & 86 & 16 & 7 \\
\hline DI (\#3) & Methanol & 1 & 74 & 1 & 0 \\
\hline
\end{tabular}

Rows are color-coded to reflect data presented in manuscript Figure $2 \mathrm{~b}$. 
Table S8: Cyprosulfamide and degradate total (Acetone/DCM + Methanol) recoveries in American River and DI water

\begin{tabular}{|c|c|c|c|c|c|}
\hline Sample & Solvent & Cyprosulfamide & $\begin{array}{c}\text { Cyprosulfamide } \\
\text { Desmethyl }\end{array}$ & $\begin{array}{c}\text { N-Cyclopropyl-4- } \\
\text { sulfamoylbenzamide }\end{array}$ & $\begin{array}{c}\mathrm{d}_{4} \text {-Imidacloprid } \\
\text { (Surrogate) }\end{array}$ \\
\hline & & \% Recovery & \% Recovery & $\%$ Recovery & \% Recovery \\
\hline $\begin{array}{l}\text { American R. } \\
(\# 1) \text { Total }\end{array}$ & $\mathrm{MeOH}$ & 75 & 38 & 82 & 62 \\
\hline $\begin{array}{l}\text { American R. } \\
(\# 2) \text { Total }\end{array}$ & $\mathrm{MeOH}$ & 62 & 26 & 71 & 55 \\
\hline $\begin{array}{l}\text { American R. } \\
\text { (\#3) Total }\end{array}$ & $\mathrm{MeOH}$ & 34 & 37 & 39 & 43 \\
\hline Average (RSD) & & $57(37)$ & $34(20)$ & $64(35)$ & $53(19)$ \\
\hline $\begin{array}{l}\text { American R. } \\
\text { (\#4) Total }\end{array}$ & $\begin{array}{c}\text { Acetone/DCM + } \\
\mathrm{MeOH}\end{array}$ & 57 & 38 & 83 & 87 \\
\hline $\begin{array}{l}\text { American R. } \\
\text { (\#5) Total }\end{array}$ & $\begin{array}{c}\text { Acetone/DCM + } \\
\mathrm{MeOH}\end{array}$ & 76 & 48 & 99 & 96 \\
\hline $\begin{array}{l}\text { American R. } \\
(\# 6) \text { Total }\end{array}$ & $\begin{array}{c}\text { Acetone/DCM + } \\
\mathrm{MeOH}\end{array}$ & 87 & 50 & 108 & 101 \\
\hline Average (RSD) & & $73(20)$ & $45(14)$ & $97(13)$ & $95(7)$ \\
\hline DI (\#1) Total & $\begin{array}{c}\text { Acetone/DCM + } \\
\mathrm{MeOH}\end{array}$ & 101 & 72 & 92 & 92 \\
\hline DI (\#2) Total & $\begin{array}{c}\text { Acetone/DCM + } \\
\mathrm{MeOH} \\
\end{array}$ & 106 & 87 & 98 & 103 \\
\hline DI (\#3) Total & $\begin{array}{c}\text { Acetone/DCM + } \\
\text { MeOH } \\
\end{array}$ & 99 & 74 & 94 & 95 \\
\hline Average (RSD) & & $102(4)$ & $78(14)$ & $95(4)$ & $96(8)$ \\
\hline
\end{tabular}

Rows are color-coded to reflect data presented in manuscript Figure $2 \mathrm{~b}$.

\section{Signal Suppression/Enhancement (SSE\%)}

Signal suppression/enhancement was evaluated by performing SPE on unspiked samples of DI and American River Water that were spiked with the analytes of interest immediately prior to analysis. These samples were compared to analytical standards by Eq. 1 below. ${ }^{5}$

$$
S S E(\%)=\frac{\text { area }(\text { spiked extract })}{\text { area }(\text { standard })} \times 100
$$

Table S9: Signal Suppression/Enhancement in DI water and American River water samples

\begin{tabular}{|c|c|c|c|c|}
\hline & Cyprosulfamide & $\begin{array}{c}\text { Cyprosulfamide } \\
\text { Desmethyl }\end{array}$ & $\begin{array}{c}\text { N-Cyclopropyl-4- } \\
\text { sulfamoylbenzamide }\end{array}$ & $\begin{array}{c}\mathrm{d}_{4} \text {-Imidacloprid } \\
\text { (Surrogate) }\end{array}$ \\
\hline & $\%$ SSE & $\%$ SSE & $\%$ SSE & $\%$ SSE \\
\hline DI Water & 95 & 96 & 95 & 102 \\
\hline American R. Water & 116 & 89 & 99 & 102 \\
\hline
\end{tabular}

Recovery of the Extraction Step $\left(\mathbf{R}_{\mathbf{E}} \%\right)$ Extraction recovery was evaluated by comparing the DI and American River extracts described above to spiked DI and American River water samples by Eq. 2 below. 5

$$
R_{E}(\%)=\frac{\text { area }(\text { spiked sample })}{\text { area }(\text { spiked extract })} \times 100
$$


Table S10: Recovery of the Extraction Step in DI water and American River water samples

\begin{tabular}{|c|c|c|c|c|}
\hline & Cyprosulfamide & $\begin{array}{c}\text { Cyprosulfamide } \\
\text { Desmethyl }\end{array}$ & $\begin{array}{c}\text { N-Cyclopropyl-4- } \\
\text { sulfamoylbenzamide }\end{array}$ & $\begin{array}{c}\mathrm{d}_{4} \text {-Imidacloprid } \\
\text { (Surrogate) }\end{array}$ \\
\hline & $\% \mathrm{R}_{\mathrm{E}}$ & $\% \mathrm{R}_{\mathrm{E}}$ & $\% \mathrm{R}_{\mathrm{E}}$ & $\% \mathrm{R}_{\mathrm{E}}$ \\
\hline DI Water & 106 & 81 & 101 & 97 \\
\hline American R. Water & 60 & 50 & 97 & - \\
\hline p-Value & 0.003 & 0.009 & 0.67 & \\
\hline
\end{tabular}

Table S11: Cyprosulfamide and degradates recoveries from American River water using preelution wash or sample cleanup procedures

\begin{tabular}{|c|c|c|c|c|}
\hline Matrix Mitigation Strategy & Cyprosulfamide & Cyprosulfamide Desmethyl & $\begin{array}{c}\text { N-Cyclopropyl-4- } \\
\text { sulfamoylbenzamide }\end{array}$ & $\begin{array}{c}\mathrm{d}_{4} \text {-Imidacloprid } \\
\text { (Surrogate) }\end{array}$ \\
\hline & \% Recovery & \% Recovery & \% Recovery & Recovery \\
\hline None (HLB, normal) & 117 & 24 & 106 & 90 \\
\hline HLB PRiME & 55 & 45 & 114 & 91 \\
\hline Water Wash & 125 & 38 & 110 & 90 \\
\hline 2\% MeOH Wash & 121 & 46 & 112 & 92 \\
\hline 5\% MeOH Wash & 118 & 39 & 101 & 91 \\
\hline Carbon Filter & 48 & 7 & 85 & 80 \\
\hline Alumina Filter & 0 & 0 & 11 & 5 \\
\hline Carbon \& Alumina Filter & 34 & 0 & & 79 \\
\hline
\end{tabular}

Table S12: Compound structures for alternate internal standards

Tebuconazole $-\mathrm{d}_{4}$




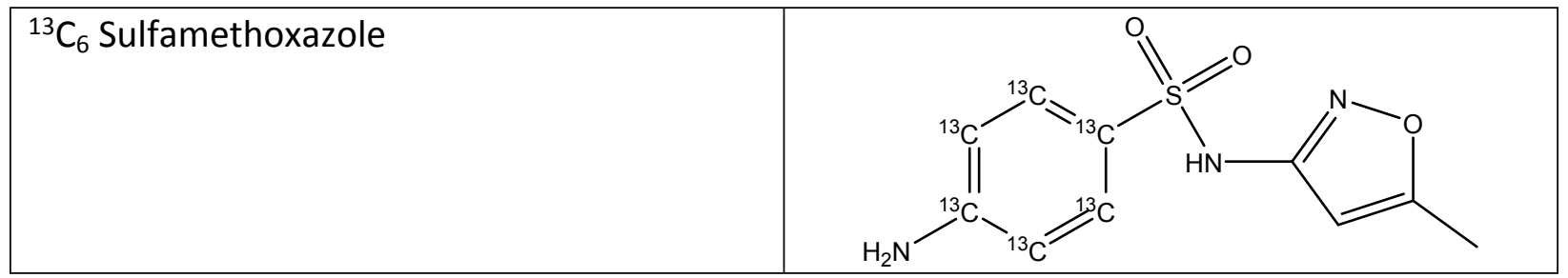

Table S13: Cyprosulfamide and degradates recoveries using alternate internal standards

\begin{tabular}{|c|c|c|c|c|}
\hline Internal Standard & Cyprosulfamide & Cyprosulfamide Desmethyl & $\begin{array}{c}\text { N-Cyclopropyl-4- } \\
\text { sulfamoylbenzamide }\end{array}$ & $\begin{array}{c}\mathrm{d}_{4} \text {-Imidacloprid } \\
\text { (Surrogate) }\end{array}$ \\
\hline & \% Recovery & \% Recovery & \% Recovery & 17 \\
\hline Sulfamethoxazole (\#1) & 66 & 43 & 61 & 19 \\
\hline Sulfamethoxazole (\#2) & 67 & 41 & 70 & 22 \\
\hline Sulfamethoxazole (\#3) & 92 & 66 & $68(10)$ & $19(12)$ \\
\hline Average (RSD) & $75(20)$ & $50(28)$ & 50 & 52 \\
\hline Myclobutanil (\#1) & 48 & 26 & 43 & 44 \\
\hline Myclobutanil (\#2) & 54 & 33 & 50 & 52 \\
\hline Myclobutanil (\#3) & 59 & 38 & $48(9)$ & $49(9)$ \\
\hline Average (RSD) & $54(10)$ & $32(18)$ & 46 & 48 \\
\hline Tebuconazole (\#1) & 45 & 26 & 39 & 40 \\
\hline Tebuconazole (\#2) & 49 & 30 & 45 & 47 \\
\hline Tebuconazole (\#3) & 54 & $31(18)$ & $43(9)$ & $45(9)$ \\
\hline Average (RSD) & $49(9)$ & & & 45 \\
\hline
\end{tabular}

Table S14: Dissolved Organic Carbon (DOC) Concentrations in surface waters used for method development

\begin{tabular}{|c|c|c|c|}
\hline Replicate & American River & Ulatis Creek & lowa River \\
\hline & DOC $(\mathrm{mg} / \mathrm{L})$ & DOC $(\mathrm{mg} / \mathrm{L})$ & DOC $(\mathrm{mg} / \mathrm{L})$ \\
\hline Sample 1 & 1.65 & 17.03 & 3.97 \\
\hline Sample 2 & 1.63 & 16.94 & 4.03 \\
\hline Sample 3 & 1.64 & 16.60 & 4.03 \\
\hline Average (RSD) & $1.64(0.74)$ & $16.86(1.30)$ & $4.01(0.81)$ \\
\hline
\end{tabular}

Table S15: Cyprosulfamide and degradate recoveries in three surface waters

\begin{tabular}{|c|c|c|c|c|c|}
\hline Source Water & $\begin{array}{c}\text { DOC } \\
(\mathrm{mg} / \mathrm{L})\end{array}$ & Cyprosulfamide & $\begin{array}{c}\text { Cyprosulfamide } \\
\text { Desmethyl }\end{array}$ & $\begin{array}{c}\text { N-Cyclopropyl-4- } \\
\text { sulfamoylbenzamide }\end{array}$ & $\begin{array}{c}\mathrm{d}_{4} \text {-Imidacloprid } \\
\text { (Surrogate) }\end{array}$ \\
\hline & & $\begin{array}{c}\text { Mean \% } \\
\text { Recovery (RSD) }\end{array}$ & $\begin{array}{c}\text { Mean \% Recovery } \\
\text { (RSD) }\end{array}$ & $\begin{array}{c}\text { Mean \% Recovery } \\
\text { (RSD) }\end{array}$ & $\begin{array}{c}\text { Mean \% } \\
\text { Recovery (RSD) }\end{array}$ \\
\hline American R. & 1.64 & $109(10)$ & $57(16)$ & $96(4)$ & $97(2)$ \\
\hline Iowa R. & 4.01 & $85(7)$ & $51(13)$ & $91(5)$ & $101(5)$ \\
\hline Ulatis Cr. & 16.86 & $80(17)$ & $38(9)$ & $98(3)$ & $98(5)$ \\
\hline
\end{tabular}


Table S16: Cyprosulfamide and degradate recovery in surface waters of varying DOC content

\begin{tabular}{|c|c|c|c|c|}
\hline Sample & Cyprosulfamide & $\begin{array}{c}\text { Cyprosulfamide } \\
\text { Desmethyl } \\
\text { \% Recovery }\end{array}$ & $\begin{array}{c}\text { N-Cyclopropyl-4- } \\
\text { sulfamoylbenzamide } \\
\text { \% Recovery }\end{array}$ & $\begin{array}{c}\mathrm{d}_{4} \text {-Imidacloprid } \\
\text { (Surrogate) } \\
\text { \% Recovery }\end{array}$ \\
\hline $\begin{array}{c}\text { American R. (\#1) } \\
\text { Total }\end{array}$ & 102 & 49 & 94 & 97 \\
\hline $\begin{array}{c}\text { American R. (\#2) } \\
\text { Total }\end{array}$ & 104 & 58 & 94 & 96 \\
\hline $\begin{array}{c}\text { American R. (\#3) } \\
\text { Total }\end{array}$ & 121 & 67 & 100 & 100 \\
\hline Average (RSD) & $109(10)$ & $58(15)$ & $96(3)$ & $97(2)$ \\
\hline lowa R. (\#1) Total & 81 & 50 & 87 & 100 \\
\hline lowa R. (\#2) Total & 89 & 58 & 92 & 97 \\
\hline lowa R. (\#3) Total & 78 & 44 & 83 & 92 \\
\hline Average (RSD) & $83(7)$ & $51(13)$ & $87(5)$ & 99 \\
\hline $\begin{array}{c}\text { Ulatis Creek (\#1) } \\
\text { Total }\end{array}$ & 84 & 37 & 94 & 102 \\
\hline $\begin{array}{c}\text { Ulatis Creek (\#2) } \\
\text { Total }\end{array}$ & 91 & 42 & 100 & $98(5)$ \\
\hline $\begin{array}{c}\text { Ulatis Creek (\#3) } \\
\text { Total }\end{array}$ & 65 & 35 & 100 & $98(3)$ \\
\hline Average & $80(17)$ & $38(9)$ & & \\
\hline
\end{tabular}

Simple linear regression was performed on the data using GraphPad Prism software to determine the statistical significance of the change in compound recovery with DOC concentration. Pvalues are provided in Table S17.

Table S17: Results of Simple Linear Regression (Percent Recovered vs DOC (mg/L))

\begin{tabular}{|c|c|c|c|}
\hline & Cyprosulfamide & Cyprosulfamide Desmethyl & N-cyclopropyl-4-sulamoylbenzamide \\
\hline Slope & -17.08 to 14.25 & -4.72 to 2.31 & -4.42 to 4.98 \\
\hline $\mathrm{R}^{2}$ & 0.57 & 0.95 & 0.36 \\
\hline P-value & 0.46 & 0.14 & 0.59 \\
\hline Significance & Not Significant & Not Significant & Not Significant \\
\hline
\end{tabular}


Table S18: Cyprosulfamide and degradate concentrations in Midwestern surface water and groundwater

\begin{tabular}{|c|c|c|c|c|c|c|}
\hline & & & Cyprosulfamide & $\begin{array}{c}\text { Cyprosulfamide } \\
\text { desmethyl }\end{array}$ & $\begin{array}{c}\mathrm{N} \text {-cyclopropyl-4- } \\
\text { sulfamoylbenzamide }\end{array}$ & $\begin{array}{c}\mathrm{d}_{4} \text {.Imidacloprid } \\
\text { (Surrogate) }\end{array}$ \\
\hline Sample Type & Site ID & $\begin{array}{c}\text { Date } \\
\text { Collected }\end{array}$ & Conc. (ng/L) & Conc. (ng/L) & Calc. Conc. (ng/L) & \% Recovery \\
\hline Surface Water & Site 1 & 4/19/2019 & nd & nd & nd & 110 \\
\hline Surface Water & Site 2 & $4 / 19 / 2019$ & nd & nd & nd & 111 \\
\hline Surface Water & Site 3 & $4 / 19 / 2019$ & nd & nd & nd & 108 \\
\hline Surface Water & $\begin{array}{c}\text { Site } 4 \\
\text { (Tile Sampler) }\end{array}$ & 4/18/2019 & nd & nd & 16.6 & 118 \\
\hline Surface Water & Pond & $4 / 24 / 2019$ & 21.4 & nd & 5.5 & 107 \\
\hline Groundwater & Well 1 & $4 / 24 / 2019$ & nd & nd & nd & 92 \\
\hline Groundwater & Well 2 & $4 / 24 / 2019$ & nd & nd & nd & 91 \\
\hline $\begin{array}{c}\text { QC Surface Water } \\
\text { (Field Blank) }\end{array}$ & Well 3 & 4/24/2019 & nd & nd & nd & 98 \\
\hline Groundwater & Well 3 & $4 / 24 / 2019$ & nd & nd & nd & 86 \\
\hline Groundwater & Well 4 & $4 / 24 / 2019$ & nd & nd & nd & 93 \\
\hline Groundwater & Well 5 & $4 / 24 / 2019$ & nd & nd & nd & 93 \\
\hline Surface Water & $\begin{array}{c}\text { Site } 4 \\
\text { (Tile Sampler) } \\
\end{array}$ & $6 / 2 / 2019$ & 5185.9 & 22.0 & 616.9 & 109 \\
\hline Surface Water & Site 3 & $6 / 2 / 2019$ & 480.2 & nd & 47.1 & 99 \\
\hline Surface Water & Site 2 & $6 / 2 / 2019$ & nd & nd & nd & 110 \\
\hline Surface Water & Pond & $9 / 5 / 2019$ & 4.5 & nd & nd & 113 \\
\hline $\begin{array}{c}\text { QC Surface Water } \\
\text { (Replicate) }\end{array}$ & Pond & 9/5/2019 & 3.7 & nd & nd & 101 \\
\hline Groundwater & W-1 & 9/5/2019 & nd & nd & nd & 91 \\
\hline Groundwater & $W-2$ & $9 / 5 / 2019$ & nd & nd & nd & 94 \\
\hline Groundwater & $W-3$ & $9 / 5 / 2019$ & nd & nd & nd & 83 \\
\hline Groundwater & W-4 & $9 / 5 / 2019$ & nd & nd & nd & 91 \\
\hline Groundwater & $W-5$ & $9 / 5 / 2019$ & nd & nd & nd & 94 \\
\hline $\begin{array}{l}\text { QC Groundwater } \\
\text { (Replicate) }\end{array}$ & W-5 & $9 / 5 / 2019$ & nd & nd & nd & 94 \\
\hline Surface Water & Site 3 & $9 / 28 / 2019$ & nd & nd & 1.9 & 92 \\
\hline Surface Water & Site 1 & $9 / 28 / 2019$ & nd & nd & nd & 88 \\
\hline $\begin{array}{c}\text { QC Surface Water } \\
\text { (Field Blank) }\end{array}$ & Site 2 & $9 / 28 / 2019$ & nd & nd & nd & 88 \\
\hline Surface Water & Site 2 & 9/28/2019 & nd & nd & 3.9 & 88 \\
\hline Surface Water & $\begin{array}{c}\text { Site } 4 \\
\text { (Tile Sampler) } \\
\end{array}$ & 10/1/2019 & nd & nd & 8.2 & 100 \\
\hline Groundwater & $W-5$ & $10 / 17 / 2019$ & nd & nd & nd & 87 \\
\hline Groundwater & W-4 & $10 / 17 / 2019$ & nd & nd & nd & 100 \\
\hline Groundwater & $W-3$ & $10 / 17 / 2019$ & nd & nd & nd & 96 \\
\hline Groundwater & $W-2$ & $10 / 17 / 2019$ & nd & nd & nd & 87 \\
\hline Groundwater & W-1 & $10 / 17 / 2019$ & nd & nd & nd & 107 \\
\hline $\begin{array}{c}\text { QC Groundwater } \\
\text { (Field Blank) }\end{array}$ & Pond & $10 / 17 / 2019$ & nd & nd & nd & 98 \\
\hline Surface Water & Pond & $10 / 17 / 2019$ & nd & nd & nd & 93 \\
\hline Surface Water & $\begin{array}{c}\text { Site } 4 \\
\text { (Tile Sampler) } \\
\end{array}$ & $10 / 26 / 2019$ & 9.6 & nd & 348.4 & 122 \\
\hline Surface Water & $\begin{array}{l}\text { Site } 5 \text { (Runoff } \\
\text { Sampler) }\end{array}$ & $10 / 26 / 2019$ & 16.0 & nd & 31.1 & 128 \\
\hline Surface Water & Site 1 & $10 / 27 / 2019$ & nd & 7.3 & nd & 102 \\
\hline
\end{tabular}




\begin{tabular}{|c|c|c|c|c|c|c|}
\hline Surface Water & Site 2 & $10 / 27 / 2019$ & nd & nd & nd & 99 \\
\hline Surface Water & Site 3 & $10 / 27 / 2019$ & nd & 3.7 & 46.6 & 101 \\
\hline $\begin{array}{c}\text { Surface Water } \\
\text { (Replicate) }\end{array}$ & Site 3 & $10 / 27 / 2019$ & nd & 4.6 & 49.7 & 112 \\
\hline
\end{tabular}

\section{References}

(1) United States Geological Survey. Water Quality Samples for the Nation https://nwis.waterdata.usgs.gov.

(2) IIHR Hydroscience and Engineering; U.S. Geological Survey. Iowa Water Quality Information System Iowa WQIS https://iwqis.iowawis.org/ (accessed Dec 10, 2020).

(3) Iowa Department of Natural Resources. Mapping and GIS https:/www.iowadnr.gov/Conservation/Mapping-GIS (accessed Dec 10, 2020).

(4) Robertson-Bryan, I.; City of Vacaville, C. Use Atainability Analysis for Municipal and Domestic Supply (MUN) Use in Segments of New Alamo Creek and Ulatis Creek, Solano County, California; 2007.

(5) Steiner, D.; Krska, R.; Malachová, A.; Taschl, I.; Sulyok, M. Evaluation of Matrix Effects and Extraction Efficiencies of LC-MS/MS Methods as the Essential Part for Proper Validation of Multiclass Contaminants in Complex Feed. J. Agric. Food Chem. 2020, 68 (12), 3868-3880. https://doi.org/10.1021/acs.jafc.9b07706. 